\title{
Nonsurgical Management of Tooth with Apical Resorption
}

\author{
${ }^{1}$ Athira A Hari, ${ }^{2}$ Praveena Geetha, ${ }^{3}$ Neethu Rajeev, ${ }^{4} \mathrm{CS}$ Deviprasad
}

\begin{abstract}
Usually external tooth resorption follows trauma to periodontal ligament. Based on clinical and histological manifestations, it can be classified into five categories. Due to the alkaline $\mathrm{pH}$, calcium hydroxide has long been used as an intracanal medicament to slow down the resorption process. Both external and internal resorptions can also occur on the same tooth, making the management more complex. This case report presents the management of a tooth with apical resorption. Apical resorption can be managed both surgically and nonsurgically. In this case, as the tooth itself has created an apical barrier, it was decided not to break the apical seal and to manage it nonsurgically. Follow-up was done for 3 and 6 months respectively.
\end{abstract}

Keywords: Calcium hydroxide, External root resorption, Osteoclast.

How to cite this article: Hari AA, Geetha P, Rajeev N, Deviprasad CS. Nonsurgical Management of Tooth with Apical Resorption. Cons Dent Endod J 2016;1(2):55-58.

\section{Source of support: Nil}

Conflict of interest: None

\section{INTRODUCTION}

Tooth resorption is usually asymptomatic and detected on routine radiographs. The etiological factors of resorption are vague; diagnosis is like educated guesses; and often the treatment does not prevent the rapid progression to dental tissues. Even the diagnostic tool has limitation, because resorption on buccal or lingual surface of tooth usually cannot be seen until 20 to $40 \%$ of tooth structure has been demineralized. ${ }^{1}$ Since the etiological factors, diagnosis, treatment, and prognosis differ for various types of resorption defects, the practitioners must be able to diagnose clinically or radiologically, distinguish between external and internal resorption, and instigate appropriate treatment. The most common causes of external resorption include trauma, periapical infection, and excess orthodontic force. ${ }^{2}$

\footnotetext{
${ }^{1,3,4}$ Postgraduate Student, ${ }^{2}$ Professor

${ }^{1-4}$ Department of Conservative Dentistry and Endodontics Azeezia College of Dental Sciences and Research, Kollam Kerala, India

Corresponding Author: Athira A Hari, Postgraduate Student Department of Conservative Dentistry and Endodontics, Azeezia College of Dental Sciences and Research, Kollam, Kerala, India Phone: +918547864854, e-mail: drathirahari@gmail.com
}

The treatment of external resorption begins by the removal of necrotic pulpal tissue to arrest the resorptive process. The aim is to remove or destroy bacteria to allow healing to take place in the periradicular space. Calcium hydroxide not only destroys the bacteria but also limits the osteoclast activity. A combination of calcium hydroxide and chlorhexidine is an effective intracanal medicament in chronic periapical lesions. ${ }^{3}$ This combination is effective against many resistant strains of bacteria and fungi. However, long-term usage of calcium hydroxide as intracanal medicament may cause weakening of the root structure in immature teeth.

Pulp necrosis after trauma causes discoloration of crown. Nonvital bleaching after root canal therapy restores the normal color of tooth. When the tooth discolors again after bleaching, other restorative procedures like crown become necessary. This case report discusses conservative management of a discolored tooth with external root resorption.

\section{CASE REPORT}

A 16-year-old female reported to the Department of Conservative Dentistry and Endodontics, Azeezia College of Dental Sciences and Research with the chief complaint of pus discharge and discoloration of her upper front tooth. The patient gave history of trauma of her upper front tooth 8 years back. She had discoloration of tooth for 5 years and pus discharge for the last 2 weeks. No history of pain, mobility, or swelling. No relevant past medical history was reported. Clinical examination revealed greyish discoloration of tooth 11 and a sinus opening in relation to attached gingiva with active pus discharge (Fig. 1).

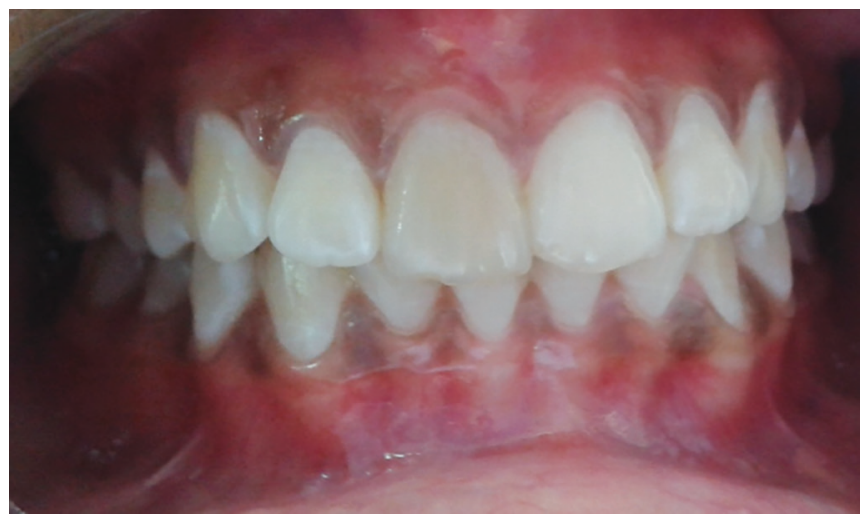

Fig. 1: Clinical picture showing grayish discoloration and sinus opening in relation to tooth 11 
The sinus tract was traced using gutta-percha point. There was no mobility or pocket formation on that tooth.

\section{Vitality Test revealed the Tooth was Nonvital}

Intraoral periapical (IOPA) of tooth 11 (Fig. 2) showed a wide root canal extending from orifice till the middle third. In the middle third of root, it appeared like an apical closure followed by radiolucency indicating resorption. Lamina dura could be traced on root surface from coronal to middle third and there is discontinuity at the apical region.

Case was diagnosed as chronic periapical abscess with apical resorption. As the tooth itself has created an apical barrier, it was decided not to break that apical seal. Nonsurgical root canal treatment with the use of calcium hydroxide as intracanal medicament and bleaching was planned for treatment. The teeth was isolated, an access cavity was made, working length was established till the calcific barrier formation, and shaping and cleaning was completed with copious irrigation with $2.5 \% \mathrm{NaOCl}$,

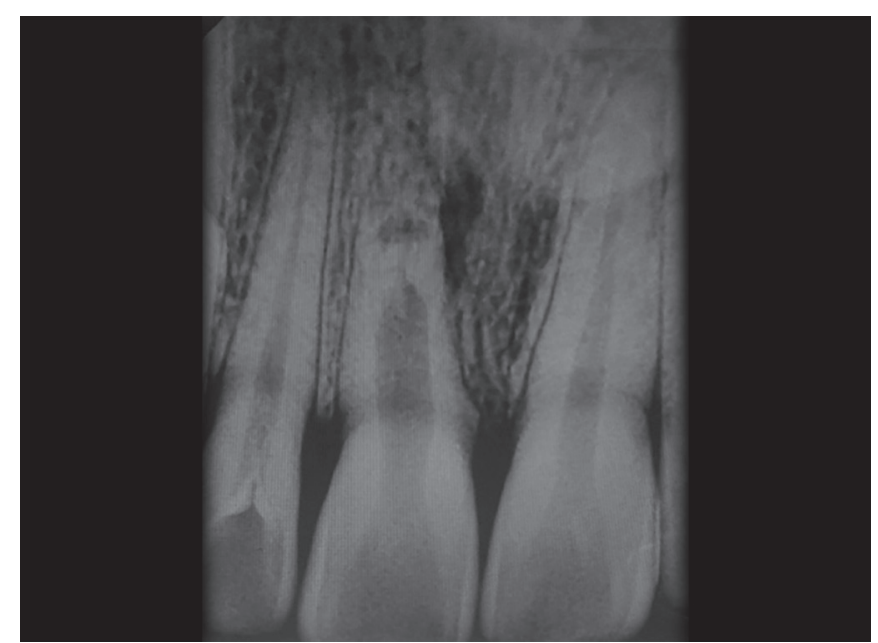

Fig. 2: Preoperative radiograph of maxillary right central incisor

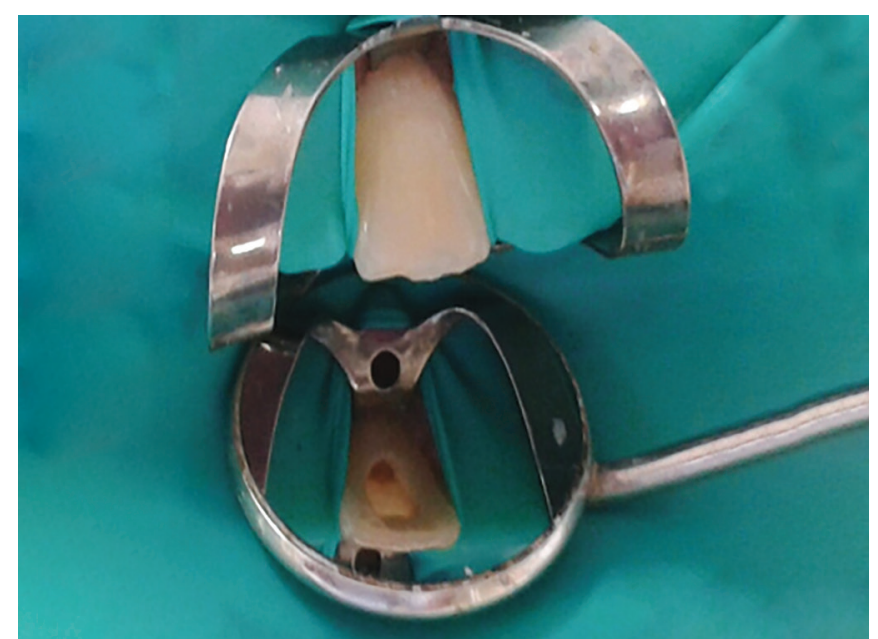

Fig. 4: Nonvital tooth bleaching of 11 saline, and $2 \%$ chlorhexidine. Care had been taken not to break the calcific barrier. Aqueous mixture of calcium hydroxide was used as an intracanal medicament $(2 \%$ chlorhexidine was used as vehicle for calcium hydroxide powder). Two weeks later the patient returned asymptomatic and the sinus was healed completely. Tooth was flushed with $10 \mathrm{~mL}$ of $2.5 \% \mathrm{NaOCl}$, saline, and $2 \%$ chlorhexidine solution. Master cone selected and obturation was done using cold lateral compaction technique till the calcific barrier (Fig. 3) using gutta-percha points (master cone and accessory cones) and zinc oxide eugenol sealer. After 2 weeks nonvital tooth bleaching was performed using sodium perborate and hydrogen peroxide (Fig. 4). A well-placed rubber dam was tightly adapted around the cervical areas of teeth. For barrier placement, epithelial attachment was determined from incisal edge using three periodontal probings from labial, mesial, and distal side. Then glass ionomer cement was placed as internal barrier $1 \mathrm{~mm}$ incisal to the epithelial attachment. After bleaching, the grayish discoloration had reduced (Fig. 5) and it was esthetically pleasing to the patient.

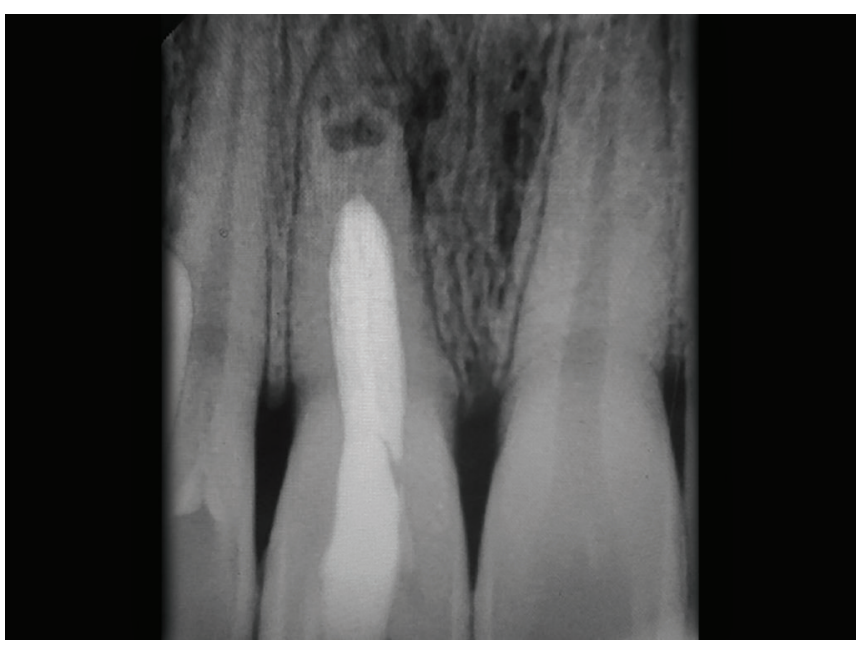

Fig. 3: Intraoral periapical of tooth 11 after obturation

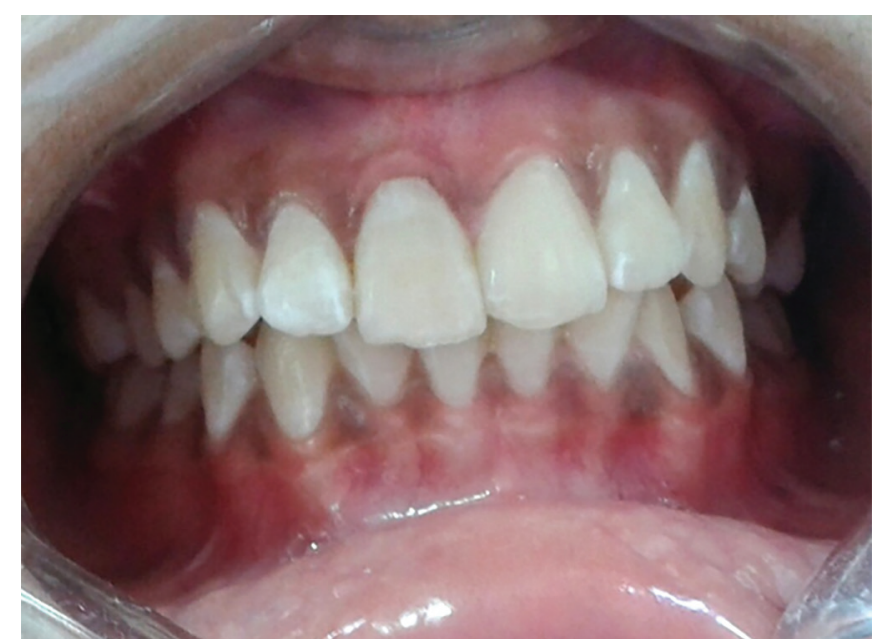

Fig. 5: Postoperative clinical picture after bleaching 


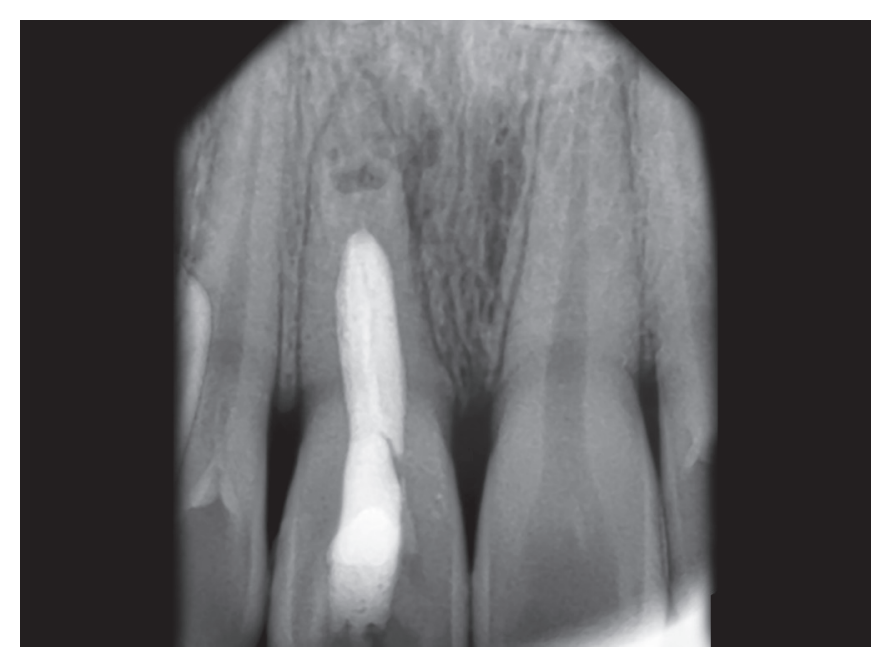

Fig. 6: Intraoral periapical of tooth 11, 3 months after obturation. The radiolucency had reduced

The patient was reviewed after 3 and 6 months. There was no pain, mobility, pus discharge, or tenderness to percussion on that tooth on follow-up clinical examination. The IOPA of 11 after 3 months showed that the radiolucency has been reduced (Fig. 6) and after 6 months, there was complete resolution of apical radiolucency with traceable lamina dura around apex (Fig. 7).

\section{DISCUSSION}

External root resorption is a progressive and destructive loss of tooth structure, initiated by a mineralized or denuded area of the root surface. The process of tooth resorption involves an elaborate interaction among inflammatory cells, resorbing cells, and hard tissue structures. It is a fact that a permanent tooth, throughout life when placed in an environment of alveolar bone surrounded by very active osteoblasts and osteoclasts, was not approached by any of these two cell lines (under normal conditions). ${ }^{4,5}$ Usually, external root resorption is difficult to predict, diagnose, and treat. The most common type of external root resorption is inflammatory root resorption. Usually it occurs following traumatic injury, chronic infection of the pulp, or periodontal structures or orthodontic tooth movement. ${ }^{6}$

The treatment goal in the external apical root resorption is to remove or destroy bacteria to allow healing to take place in the periradicular space. The most common cause of external root resorption is trauma, particularly in cases, where the injury results in pulpal necrosis and damage to the root surface, leaving dentinal tubules exposed. This creates a communication between the internal and external surfaces of the root. Bacteria, bacterial byproducts, and tissue breakdown products from within the root canal system stimulate inflammation in the adjacent

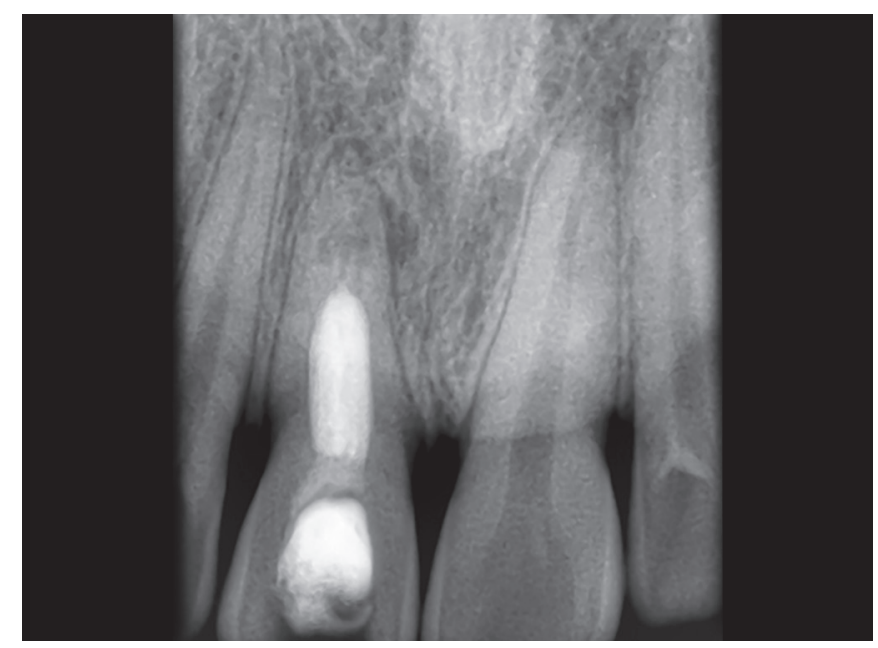

Fig. 7: Intraoral periapical of 11, 6 months after obturation. The radiolucency had reduced. Osseous formation noted at apex

periodontal tissues and lead to aggressive and progressive inflammatory resorption of the root. Nonsurgical root canal therapy combined with a calcium hydroxide dressing was recommended as treatment by Andreasen. Calcium hydroxide as an intracanal medicament best destroys the bacteria. ${ }^{7}$ The human body is continually working to keep the internal environment in a condition of homeostasis.

The host immune system responds to this bacterial challenge through an array of coordinated but complex process that are designed to eliminate the initiating agent and return the site to a state of homeostasis. In the present case, robustness of host immune response might have formed an apical barrier to counter the resorption. Treatment of external root resorption is dependent on the etiology. In case where the resorption is due to pulpal necrosis and periodontal injury, nonsurgical pulp space therapy is performed with the use of calcium hydroxide as an interim medicament. Complete chemomechanical preparation is considered as an essential step in root canal disinfection. However, total elimination of bacteria is difficult to accomplish. By remaining in the canal between appointments, intracanal medicament may help to eliminate surviving bacteria. ${ }^{7}$

Recently, interest has been focused on the effectiveness of mixture of calcium hydroxide and chlorhexidine as intracanal medicament. Chlorhexidine increases the antibacterial effectiveness of calcium hydroxide paste. ${ }^{8,9}$ Chlorhexidine combined with calcium hydroxide had greater antimicrobial activity than calcium hydroxide mixed with water. It can completely disinfect the root canal system by achieving a $\mathrm{pH}$ of 12.8 . This combination will be effective against Enterococcus faecalis also. ${ }^{10}$

In this case report, the IOPA of tooth 11 in 6 months follow-up showed complete resolution of apical radiolucency with traceable lamina dura around apex. Apical 
one-third of the root has undergone a remodeling, suggestive of complete healing.

\section{CONCLUSION}

An inflammatory external root resorption requires endodontic treatment to remove the necrotic tissue and arrest the progression of resorption. In the present case nonsurgical pulp space therapy with calcium hydroxide and chlorhexidine as intracanal medicament had effectively arrested the external root resorption and a favorable healing had taken place.

\section{REFERENCES}

1. Andreasen J, Bakland L. Pathologic tooth resorption. In: Ingle J, Bakland L, Baumgartner J, editors. Ingle's endodontics. 6th ed. Canada: BC Decker; 2008. p. 1358-1382.

2. Armas JM, Savarrio L, Brocklebank LM. External apical root resorption: two case reports. Int Endod J 2008 Nov;41(11): 997-1004.
3. Mohammadi Z, Abott PV. The properties and application of chlorhexidine in endodontics. Int Endod J 2009 Apr;42(4): 288-302.

4. Gunraj MN. Dental root resorption. Oral Surg Oral Med Oral Pathol 1999 Dec;88(6):647-654.

5. Fuss Z, Tsesis I, Lin S. Root resorption-diagnosis, classification and treatment choices based on stimulation factors. Dental Traumatol 2003 Aug;19(4):175-182.

6. Ne RF, Witherspoon DE, Gutmann JL. Tooth resorption. Quintessence Int 1999 Jan;30(1):9-25.

7. Siqueira JF Jr, Lopes HP. Mechanism of antimicrobial activity of calcium hydroxide: a critical review. Int Endod J 1999 Sep;32(5):361-369.

8. Fava LR, Saunders WP. Calcium hydroxide pastes: classification and clinical indications. Int Endod J 1999 Aug;32(4): 257-282.

9. Siqueira JF Jr, Paiva SS, Rocas IN. Reduction in the cultivable bacterial populations in infected root canals by a chlorhexidine based antimicrobial protocol. J Endod 2007 May;33(5):541-547.

10. Stuart CH, Schwartz SA, Beeson TJ, Owatz CB. Enterococcus faecalis: its role in root canal treatment failure and current concepts in retreatment. J Endod 2006 Feb;32(2):93-98. 